\title{
ON THE MODEL EQUATIONS WHICH DESCRIBE NONLINEAR WAVE MOTIONS IN A ROTATING FLUID ${ }^{1,2}$
}

\author{
BY
}

\author{
JONG UHN KIM
}

\begin{abstract}
This paper concerns mathematical aspects of the two model equations describing nonlinear wave motions in a rotating fluid. We establish local existence of solutions and show that singularities occur in a finite time under certain hypotheses. We also show that these equations admit nonconstant travelling wave solutions.
\end{abstract}

0. Introduction. The purpose of this paper is to report some results on qualitative properties of the two model equations which arise in the theory of long waves in a rotating fluid:

$$
\begin{gathered}
u_{t}+\alpha u_{x}+u u_{x}+A \frac{\partial^{3}}{\partial x^{3}} \int_{-\infty}^{\infty} \frac{u(\eta, t)}{\sqrt{(x-\eta)^{2}+B^{2}}} d \eta=0 \\
u_{t}+\alpha u_{x}+u u_{x}-A \frac{\partial}{\partial t} \frac{\partial^{2}}{\partial x^{2}} \int_{-\infty}^{\infty} \frac{u(\eta, t)}{\sqrt{(x-\eta)^{2}+B^{2}}} d \eta=0
\end{gathered}
$$

Equation (0-1) was derived by Leibovich [9]. This equation describes axially symmetric long wave motions of small amplitude in inviscid, incompressible, rotating fluids which are radially infinite. Here $A \neq 0, B>0$ and $\alpha$ are real constants, $t$ is the time variable and $x$ the axial coordinate. When $r$ denotes the radial coordinate, the wave disturbance stream function $\psi(r, x, t)$ is assumed to be of the form $\psi(r, x, t)=\varepsilon \phi(r) u(x, t)$ and $u(x, t)$ satisfies (0-1); for details, see [9]. Equation $(0-2)$ is obtained from $(0-1)$ by exploiting the zero-order equivalence of $\partial / \partial x$ and $-\partial / \partial t$ (see [2]).

It is interesting to compare equation (0-1) with Burger's equation (without dissipation) and with the $\mathrm{K}-\mathrm{dV}$ equation:

$$
u_{t}+\alpha u_{x}+u u_{x}=0
$$

Received by the editors August 23, 1983 and, in revised form, February 13, 1984. This result was presented at the 90th annual meeting of the AMS at Louisville, January 27, 1984.

1980 Mathematics Subject Classification. Primary 35Q20, 76B15, 35B10, 35B32, 35B40.

Key words and phrases. Nonlinear wave motion, rotating fluid, local existence of solution, formation of singularities, periodic travelling wave solution.

${ }^{1}$ The work for this paper was done while the author was at the Mathematics Research Center, University of Wisconsin-Madison.

${ }^{2}$ This research was supported by the United States Army under Contract No. DAAG-80-C-0041 and by the National Science Foundation under Grant No. MCS-7927062, Mod. 2. 


$$
u_{t}+\alpha u_{x}+u u_{x}+u_{x x x}=0 .
$$

Equation ( $0-3)$ is obtained from $(0-1)$ by deleting the dispersion term, and equation $(0-4)$ has a dispersion term which is stronger than that of $(0-1)$; see [2]. Now we consider some basic mathematical questions associated with an evolution equation:

(Q1) Does there exist a unique local solution of the initial value problem in a reasonably smooth function space?

(Q2) Can this local solution be defined globally in time in the same function space?

(Q3) Does the equation admit a nonconstant continuous travelling wave solution?

The main results of this paper are the answers to these questions; see Theorems 1.1, 2.1, 3.4 and 3.6. Actually we discuss more general equations which include (0-1) and $(0-2)$. The qualitative theory for equation $(0-4)$ has been developed by many authors and the results are well known. With regard to the above questions, we compare the equations as follows:

\begin{tabular}{|c|c|c|c|}
\hline & $(0-1),(0-2)$ & $(0-3)$ & $(0-4)$ \\
\hline$(Q 1)$ & Yes & Yes & Yes \\
\hline$(Q 2)$ & No & No & Yes \\
\hline (Q3) & Yes & No & Yes \\
\hline
\end{tabular}

This summary will be explained in detail in the subsequent sections.

Notations. The standard notations $\partial_{t}, \partial_{x}, f_{t}, f_{x}$ are used for $\partial / \partial t, \partial / \partial x, \partial f / \partial t$, $\partial f / \partial x$, respectively. For a given function $g$, its Fourier transform is defined (as usual) by

$$
\hat{g}(\xi)=\frac{1}{\sqrt{2 \pi}} \int_{-\infty}^{\infty} g(x) e^{-i x \xi} d x .
$$

$H^{s}, s \geqslant 0$, stands for the set of all real-valued functions $g$ in $L^{2}(R)$ such that

$$
\int_{-\infty}^{\infty}\left(1+|\xi|^{2}\right)^{s}|\hat{g}(\xi)|^{2} d \xi<\infty
$$

and the norm $\|\cdot\|_{H^{s}}$ is taken to be

$$
\|g\|_{H^{s}}^{2}=\int_{-\infty}^{\infty}\left(1+|\xi|^{2}\right)^{s}|\hat{g}(\xi)|^{2} d \xi
$$

$H^{1}(0, p)$ denotes the set of all real-valued functions in $L^{2}(0, p)$ whose first order derivative is also in $L^{2}(0, p)$. The elements of $C_{0}(R)$ are continuous functions in $R$ which vanish at infinity.

1. Local existence of solutions. Under some physical hypotheses, the general model equations for long waves are given by either

$$
u_{t}+\alpha u_{x}+u u_{x}+\partial_{x} \mathbf{P}_{1} u=0
$$

or

$$
u_{t}+\alpha u_{x}+u u_{x}+\partial_{t} \mathbf{P}_{2} u=0
$$


where $\mathbf{P}_{1}$ and $\mathbf{P}_{2}$ are pseudo-differential operators. In the derivation of such equations, the operators $\mathbf{P}_{i}, i=1,2$, are usually determined from the dispersion relation in terms of their symbols $\hat{\mathbf{P}}_{i}(\xi), i=1,2$ (see $\left.[1,2,9]\right)$. Hence, we assume that the symbols $\hat{\mathbf{P}}_{i}(\xi), i=1,2$, are given in advance to define the operators $\mathbf{P}_{i}, i=1,2$. For $(0-1),(0-2)$, the corresponding operators are given by

$$
-\hat{\mathbf{P}}_{1}(\xi)=\hat{\mathbf{P}}_{2}(\xi)=A \sqrt{2 / \pi} \xi^{2} K_{0}(B|\xi|),
$$

where $K_{0}(\cdot)$ is the modified Bessel function of the second kind of order zero. In this section, we establish the local existence of solutions to (1-1), (1-2) in the case where the symbols $\hat{\mathbf{P}}_{i}(\xi), i=1,2$, satisfy the following conditions:

$$
\hat{\mathbf{P}}_{i}(\xi), i=1,2 \text {, are even, real-valued functions; }
$$

$$
\xi \hat{\mathbf{P}}_{i}(\xi) \in L^{\infty}, i=1,2 \text {; }
$$

(1-6) for some $-1<\delta<M<\infty, \delta \leqslant \hat{\mathbf{P}}_{2}(\xi) \leqslant M$ holds for almost all $\xi \in R$.

Since $K_{0}(|\xi|)$ behaves like $-\log |\xi|$ for small $\xi$ and like $\sqrt{\pi / 2|\xi|} e^{-|\xi|}$ for large $\xi$, it is easy to see that (1-4), (1-5) are satisfied by (1-3), and (1-6) is satisfied when

$$
A>\sqrt{\frac{\pi}{2}} \frac{-1}{\max _{\xi \in R} \xi^{2} K_{0}(B|\xi|)} .
$$

Now we state the local existence theorem.

THEOREM 1.1. Suppose conditions (1-4) to (1-6) hold. Let $s>3 / 2$ and $u_{0}(x) \in H^{s}$. Then there is a positive number $T$ depending on $\left\|u_{0}\right\|_{H^{s}}$ such that (1-1), (1-2) have unique solutions in $C\left([0, T) ; H^{s}\right) \cap C^{1}\left([0, T) ; H^{s-1}\right)$ satisfying $u(x, 0)=u_{0}(x)$.

Proof. Let $s>3 / 2$ be given. By virtue of (1-4) and (1-5), it is obvious that $f \rightarrow \partial_{x} \mathbf{P}_{1} f$ is a continuous mapping from $H^{s}$ into itself satisfying:

$$
\begin{gathered}
\left\|\partial_{x} \mathbf{P}_{1} f\right\|_{H^{s}} \leqslant\left\|\xi \hat{\mathbf{P}}_{1}(\xi)\right\|_{L^{\infty}}\|f\|_{H^{s}}, \quad \text { for all } f \in H^{s}, \\
\left\|\partial_{x} \mathbf{P}_{1} f-\partial_{x} \mathbf{P}_{1} g\right\|_{L^{2}} \leqslant\left\|\xi \hat{\mathbf{P}}_{1}(\xi)\right\|_{L^{\infty}}\|f-g\|_{L^{2}}, \quad \text { for all } f, g \in H^{s} .
\end{gathered}
$$

Following the notation in Kato [6], we take $X=L^{2}, Y=H^{s}$. Then, by means of $(1-7),(1-8)$, we find that $(1-1)$ is a special version of Example 8.1 in [6], so we can employ Kato's result directly to obtain a unique solution in $C\left([0, T) ; H^{s}\right) \cap$ $C^{1}\left([0, T) ; H^{s-1}\right)$ for some $T>0$. Next we rewrite $(1-2)$ in the form

$$
u_{t}+\alpha u_{x}+u u_{x}+\mathbf{P}_{3}\left(\alpha u_{x}+u u_{x}\right)=0,
$$

where $\hat{\mathbf{P}}_{3}(\xi)=-\hat{\mathbf{P}}_{2}(\xi) /\left(1+\hat{\mathbf{P}}_{2}(\xi)\right)$. By taking account of the hypotheses on $\hat{\mathbf{P}}_{2}(\xi)$, it can be easily shown that if $u \in C\left([0, T) ; H^{s}\right) \cap C^{1}\left([0, T) ; H^{s-1}\right), s>3 / 2$, then $(1-2)$ and (1-9) are equivalent, that is, a solution of (1-9) in the above function space is also a solution of (1-2) and vice versa. Conditions (1-4) to (1-6) imply that $\xi \hat{\mathbf{P}}_{3}(\xi) \in L^{\infty}$ and $f \rightarrow \mathbf{P}_{3} f_{x}$ is a continuous mapping from $H^{s}$ into itself. The mapping $(f, g) \rightarrow f g$ is a continuous bilinear mapping from $H^{\sigma} \times H^{\sigma}$ into $H^{\sigma}$, provided $\sigma>1 / 2$, and hence, it follows that for all $f \in H^{s}$,

$$
\left\|\mathbf{P}_{3}\left(\alpha f_{x}+f f_{x}\right)\right\|_{H^{s}} \leqslant \mathbf{M}\left\|\xi \hat{\mathbf{P}}_{3}(\xi)\right\|_{L^{\infty}}\left(\|f\|_{H^{s}}+\|f\|_{H^{s}}^{2}\right),
$$


and for all $f, g \in H^{s}$,

$$
\begin{aligned}
& \left\|\mathbf{P}_{3}\left(\alpha f_{x}+f f_{x}\right)-\mathbf{P}_{3}\left(\alpha g_{x}+g g_{x}\right)\right\|_{L^{2}} \\
& \quad \leqslant \mathbf{M}\left\|\xi \hat{\mathbf{P}}_{3}(\xi)\right\|_{L^{\infty}}\left\{\|f+g\|_{H^{s}}\|f-g\|_{L^{2}}+\|f-g\|_{L^{2}}\right\},
\end{aligned}
$$

where $M>0$ depends only on $\alpha$. By taking $X=L^{2}, Y=H^{s}$, and using (1-10), (1-11), it is easily seen that (1-9) is also a special version of Example 8.1 in [6], and the local existence of solutions to (1-9) follows immediately.

2. Formation of singularities. Since local solutions have been obtained in $C\left([0, T) ; H^{s}\right), s>3 / 2$, we shall examine the possibility of extending a local solution to a global solution. It turns out that singularities develop in local solutions under the additional assumptions and, thus, a local solution cannot be extended globally in time in general. In this section we assume not only (1-4) to (1-6), but also the following conditions:

$$
\begin{gathered}
\xi^{2} \hat{\mathbf{P}}_{1}(\xi) \in L^{2} \\
\xi^{2} \hat{\mathbf{P}}_{2}(\xi) \in L^{1} \cap L^{2} .
\end{gathered}
$$

Obviously, (2-1), (2-2) are satisfied by (1-3). The main result of this section is:

THEOREM 2.1. Under the above assumptions the local solutions of (1-1), (1-2) in $C\left([0, T) ; H^{s}\right), s>3 / 2$, cannot be extended globally in time in the same function space if the initial function $u_{0}(x) \in H^{s}$ satisfies:

$$
\begin{gathered}
\lambda / \mu^{2}<\kappa_{1}, \text { for equation }(1-1) ; \\
\left(\lambda+\lambda^{2}\right) \mu^{2}<\kappa_{2}, \text { for equation }(1-2),
\end{gathered}
$$

where $\lambda=\left\|u_{0}\right\|_{L^{2}}>0, \mu=-\min _{x \in R} u_{0 x}(x)$ and $\kappa_{1}\left(\right.$ resp. $\left.\kappa_{2}\right)$ is a positive constant depending only on $\mathbf{P}_{1}$ (resp. $\mathbf{P}_{2}$ ). As a consequence, we have

THEOREM 2.2. Let $s_{1}<3$ be given. Then, for any $\varepsilon>0$, there are initial data $u_{0}(x)$ in $H^{s_{1}}$ such that $\left\|u_{0}\right\|_{H^{s_{1}}}<\varepsilon$ and no global solution satisfying the initial condition $u(x, 0)=u_{0}(x)$ exists in $C\left([0, \infty) ; H^{s_{2}}\right)$ for any $s_{2}>3 / 2$.

Proof. We may take $3 / 2<s_{1}<3-\delta$ for some $\delta>0$. Let $f$ be any (nontrivial) function in $H^{s_{1}}$ and define $f_{\varepsilon}(x)=\varepsilon^{5 / 2-\delta} f(x / \varepsilon)$. Let $\beta=\|f(\cdot)\|_{L^{2}}, \quad r=$ $-\min _{x \in R} f_{x}(x)$ and $\rho=\|f(\cdot)\|_{H^{s 1}}$. Then

$$
\left\|f_{\varepsilon}(\cdot)\right\|_{L^{2}}=\varepsilon^{3-\delta} \beta, \quad \min _{x \in R} f_{\varepsilon x}(x)=-\varepsilon^{3 / 2-\delta} r
$$

and

$$
\left\|f_{\varepsilon}(\cdot)\right\|_{H^{s_{1}}} \leqslant 2^{s_{1} / 2} \varepsilon^{3-\delta} \beta+2^{s_{1} / 2} \varepsilon^{3-s_{1}-\delta} \rho .
$$

By taking $\varepsilon$ sufficiently small, $f_{\varepsilon}(\cdot)$ is a desired initial function satisfying $(2-3)$ and (2-4).

To prove Theorem 2.1, we need the following lemma. 
LEMMA 2.3. Suppose (1-1), (1-2) have solutions $u_{1}(x, t), u_{2}(x, t)$, respectively, in $C\left([0, T) ; H^{s}\right), s>3 / 2$, satisfying $u_{i}(x, 0)=u_{0}(x) \in H^{s}, i=1,2$. Then the following hold:

$$
\begin{gathered}
\left\|u_{1}(x, t)\right\|_{L^{2}}=\left\|u_{0}(x)\right\|_{L^{2}}, \quad \text { for all } t \in[0, T), \\
\left\|u_{2}(x, t)\right\|_{L^{2}} \leqslant \mathbf{M}\left\|u_{0}(x)\right\|_{L^{2}}, \text { for all } t \in[0, T),
\end{gathered}
$$

where $M$ is a positive constant depending only on $\mathbf{P}_{2}$.

Proof. Combined with (1-1), (1-2), $u(x, t) \in C\left([0, T) ; H^{s}\right)$ implies $u(x, t) \in$ $C^{1}\left([0, T) ; H^{s-1}\right)$. Multiplying both sides of (1-1), (1-2) by $u_{1}(x, t), u_{2}(x, t)$, respectively, and integrating over $R$, we obtain

$$
\begin{gathered}
\frac{d}{d t} \int_{-\infty}^{\infty} u_{1}^{2}(x, t) d x=0 \\
\frac{d}{d t} \int_{-\infty}^{\infty}\left(1+\hat{\mathbf{P}}_{2}(\xi)\right)\left|\hat{u}_{2}(\xi, t)\right|^{2} d \xi=0
\end{gathered}
$$

since

$$
\begin{gathered}
\int_{-\infty}^{\infty} u_{i x} u_{i} d x=0, \quad \int_{-\infty}^{\infty}\left(u_{i} u_{i x}\right) u_{i} d x=0, \quad i=1,2 \\
\int_{-\infty}^{\infty}\left(\mathbf{P}_{1} u_{1 x}\right) u_{1} d x=\int_{-\infty}^{\infty} i \xi \hat{\mathbf{P}}_{1}(\xi)\left|\hat{u}_{1}(\xi, t)\right|^{2} d \xi=0
\end{gathered}
$$

(Notice that $u_{1}$ is a real-valued function and, hence, $\left|\hat{u}_{1}(\xi, t)\right|^{2}$ is an even function of $\xi$.) $(2-5)$ is implied by (2-7) and, by making use of $(2-8)$ and (1-6), (2-6) is easily deduced.

Now we proceed to:

Proof of Theorem 2.1. With the aid of (1-4) to (1-6) and (2-1), (2-2), we can express $\mathbf{P}_{1} f_{x}$ and $\mathbf{P}_{3} f_{x}$ (defined in the preceding section) for $f \in H^{\sigma}, \boldsymbol{\sigma} \geqslant 1$, as follows:

$$
\mathbf{P}_{i} f_{x}=\int_{-\infty}^{\infty} G_{i}(x-y) f(y) d y, \quad i=1,3,
$$

where $G_{i}, i=1,3$, satisfy

$$
\begin{aligned}
G_{1} \in L^{2}, & G_{1 x} \in L^{2} ; \\
G_{3} \in L^{2} \cap C_{0}, & G_{3 x} \in L^{2} \cap C_{0} .
\end{aligned}
$$

We assume that for given nontrivial $u_{0}(x)$ in $H^{s}, s>3 / 2$, there is a global solution $u(x, t)$ of $(1-1)$ in $C\left([0, \infty) ; H^{s}\right)$ which, combined with (1-1), implies

$$
u \in C^{1}(R \times[0, \infty)) \text {. }
$$

Following the classical theory of characteristics, we solve the initial value problem for each fixed $\eta \in R$ :

$$
\begin{gathered}
d x(t, \eta) / d t=\alpha+u(x(t, \eta), t), \\
x(0, \eta)=\eta .
\end{gathered}
$$


Observing that $u(x, t)$ is uniformly bounded on $R \times[0, T]$, for each $T>0$, we can conclude that $x(t, \eta) \in C^{1}([0, \infty) \times R)$ and $x\left(t, \eta_{2}\right)>x\left(t, \eta_{1}\right)$ for all $t \geqslant 0$, provided $\eta_{2}>\eta_{1}$ (see Hale [5]). With this $x(t, \eta), u(x(t, \eta), t)$ is regarded as a function of $t$ for each $\eta$ and satisfies, for each fixed $\eta$,

$$
\frac{d}{d t} u(x(t, \eta), t)=-\int_{-\infty}^{\infty} G_{1}(x(t, \eta)-y) u(y, t) d y, \text { for all } t \geqslant 0
$$

and

$$
u(x(0, \eta), 0)=u_{0}(\eta) .
$$

Since $u_{0}(\eta) \neq 0$, there are $\mu>0$ and $\eta_{1}<\eta_{2}$ such that

$$
\left(u_{0}\left(\eta_{2}\right)-u_{0}\left(\eta_{1}\right)\right) /\left(\eta_{2}-\eta_{1}\right)=-\mu .
$$

Let us set $\omega=u_{0}\left(\eta_{1}\right)-u_{0}\left(\eta_{2}\right)>0, \eta_{2}-\eta_{1}=l>0$. From (2-16), (2-17), it follows that for all $t \geqslant 0$,

$$
\text { (2-19) } \begin{aligned}
u\left(x\left(t, \eta_{1}\right), t\right)-u\left(x\left(t, \eta_{2}\right), t\right)=u_{0}\left(\eta_{1}\right)-u_{0}\left(\eta_{2}\right) \\
-\int_{0}^{t} d \tau \int_{-\infty}^{\infty}\left\{G_{1}\left(x\left(\tau, \eta_{1}\right)-y\right)-G_{1}\left(x\left(\tau, \eta_{2}\right)-y\right)\right\} u(y, \tau) d y
\end{aligned}
$$

which, together with Lemma 2.3 and (2-12), implies

(2-20) $u\left(x\left(t, \eta_{1}\right), t\right)-u\left(x\left(t, \eta_{2}\right), t\right) \geqslant \omega-\int_{0}^{t} d \tau M_{1} \lambda\left|x\left(\tau, \eta_{1}\right)-x\left(\tau, \eta_{2}\right)\right|$,

for all $t \geqslant 0$, and

(2-21) $\left|u\left(x\left(t, \eta_{1}\right), t\right)-u\left(x\left(t, \eta_{2}\right), t\right)\right| \leqslant \omega+\int_{0}^{t} d \tau M_{1} \lambda\left|x\left(\tau, \eta_{1}\right)-x\left(\tau, \eta_{2}\right)\right|$,

for all $t \geqslant 0$, where $M_{1}=\left\|G_{1 x}\right\|_{L^{2}}$ and $\lambda=\left\|u_{0}(\eta)\right\|_{L^{2}}$. But, (2-14) and (2-15) yield

$$
\left|x\left(\tau, \eta_{1}\right)-x\left(\tau, \eta_{2}\right)\right| \leqslant l+\int_{0}^{\tau} d \zeta\left|u\left(x\left(\zeta, \eta_{1}\right), \zeta\right)-u\left(x\left(\zeta, \eta_{2}\right), \zeta\right)\right|
$$

Consequently, for all $0 \leqslant t \leqslant 1 / \mu$, we have

$$
\begin{aligned}
& \left|u\left(x\left(t, \eta_{1}\right), t\right)-u\left(x\left(t, \eta_{2}\right), t\right)\right| \\
& \quad \leqslant \omega+\int_{0}^{t} d \tau M_{1} \lambda\left\{l+\int_{0}^{\tau} d \zeta\left|u\left(x\left(\zeta, \eta_{1}\right), \zeta\right)-u\left(x\left(\zeta, \eta_{2}\right), \zeta\right)\right|\right\}
\end{aligned}
$$

(by using $\omega=l \mu$ )

$$
\begin{aligned}
& \leqslant \omega\left(1+\frac{M_{1} \lambda}{\mu^{2}}\right)+M_{1} \lambda \int_{0}^{t} d \zeta(t-\zeta)\left|u\left(x\left(\zeta, \eta_{1}\right), \zeta\right)-u\left(x\left(\zeta, \eta_{2}\right), \zeta\right)\right| \\
& \leqslant \omega\left(1+\frac{M_{1} \lambda}{\mu^{2}}\right)+M_{1} \lambda \int_{0}^{t} d \zeta\left(\frac{1}{\mu}-\zeta\right)\left|u\left(x\left(\zeta, \eta_{1}\right), \zeta\right)-u\left(x\left(\zeta, \eta_{2}\right), \zeta\right)\right| .
\end{aligned}
$$

By Gronwall's inequality we arrive at

$$
\left|u\left(x\left(t, \eta_{1}\right), t\right)-u\left(x\left(t, \eta_{2}\right), t\right)\right| \leqslant \omega\left(1+M_{1} \lambda / \mu^{2}\right) e^{M_{1} \lambda / 2 \mu^{2}},
$$


for all $0 \leqslant t \leqslant 1 / \mu$ and, hence,

$$
\begin{gathered}
M_{1} \lambda \int_{0}^{1 / \mu}\left(\frac{1}{\mu}-\zeta\right)\left|u\left(x\left(\zeta, \eta_{1}\right), \zeta\right)-u\left(x\left(\zeta, \eta_{2}\right), \zeta\right)\right| d \zeta \\
\leqslant M_{1} \lambda \omega\left(1+\frac{M_{1} \lambda}{\mu^{2}}\right) \frac{1}{2 \mu^{2}} e^{M_{1} \lambda / 2 \mu^{2}}
\end{gathered}
$$

In the mean time, substituting (2-22) into (2-20) we have, for all $0 \leqslant t \leqslant 1 / \mu$,

$$
\begin{aligned}
u\left(x\left(t, \eta_{1}\right), t\right)-u\left(x\left(t, \eta_{2}\right), t\right) & \\
& \geqslant \omega\left(1-\frac{M_{1} \lambda}{\mu^{2}}\right)-M_{1} \lambda \int_{0}^{t} d \tau \int_{0}^{\tau} d \zeta\left|u\left(x\left(\zeta, \eta_{1}\right), \zeta\right)-u\left(x\left(\zeta, \eta_{2}\right), \zeta\right)\right| \\
& =\omega\left(1-\frac{M_{1} \lambda}{\mu^{2}}\right)-M_{1} \lambda \int_{0}^{t} d \zeta(t-\zeta)\left|u\left(x\left(\zeta, \eta_{1}\right), \zeta\right)-u\left(x\left(\zeta, \eta_{2}\right), \zeta\right)\right| \\
& \geqslant \omega\left(1-\frac{M_{1} \lambda}{\mu^{2}}\right)-M_{1} \lambda \int_{0}^{1 / \mu} d \zeta\left(\frac{1}{\mu}-\zeta\right)\left|u\left(x\left(\zeta, \eta_{1}\right), \zeta\right)-u\left(x\left(\zeta, \eta_{2}\right), \zeta\right)\right|,
\end{aligned}
$$

by using (2-25)

$$
\geqslant \omega\left\{1-\frac{M_{1} \lambda}{\mu^{2}}-\frac{M_{1} \lambda}{2 \mu^{2}}\left(1+\frac{M_{1} \lambda}{\mu^{2}}\right) e^{M_{1} \lambda / 2 \mu^{2}}\right\} .
$$

Now we choose $\kappa>0$ such that

$$
\begin{gathered}
M_{1}\left\{\kappa+\frac{1}{2} \kappa\left(1+M_{1} \kappa\right) e^{M_{1} \kappa / 2}\right\} \leqslant 1 / 2, \\
M_{1} \kappa e^{M_{1} \kappa / 2}<1 / 2 .
\end{gathered}
$$

Then

$$
\lambda / \mu^{2} \leqslant \kappa
$$

implies

(2-30) $\quad u\left(x\left(t, \eta_{1}\right), t\right)-u\left(x\left(t, \eta_{2}\right), t\right) \geqslant \frac{1}{2} \omega$, for all $0 \leqslant t \leqslant 1 / \mu$.

For the remainder of the proof, we assume (2-29) holds. We proceed to estimate $x\left(t, \eta_{2}\right)-x\left(t, \eta_{1}\right)$. Combining (2-14) to (2-17), it is apparent that

$$
x(t, \eta)=\eta+\int_{0}^{t} d \tau\left\{\alpha+u_{0}(\eta)-\int_{0}^{\tau} d \zeta \int_{-\infty}^{\infty} G_{1}(x(\zeta, \eta)-y) u(y, \zeta) d y\right\},
$$

for all $t \geqslant 0, \eta \in R$, and thus,

$$
\begin{aligned}
x\left(t, \eta_{2}\right)-x\left(t, \eta_{1}\right)=l-\omega t-\int_{0}^{t} d \tau \int_{0}^{\tau} d \zeta\left\{\int_{-\infty}^{\infty}\right. & \left(G_{1}\left(x\left(\zeta, \eta_{2}\right)-y\right)\right. \\
& \left.\left.-G_{1}\left(x\left(\zeta, \eta_{1}\right)-y\right)\right) u(y, \zeta) d y\right\} .
\end{aligned}
$$


Set $f(t)=x\left(t, \eta_{2}\right)-x\left(t, \eta_{1}\right)$; then $f(t)>0$ for all $t \geqslant 0$. From (2-32), we derive the estimate:

$$
\begin{aligned}
f(t) & \leqslant l-\omega t+\int_{0}^{t} d \tau\left\{M_{1} \lambda \int_{0}^{\tau} f(\zeta) d \zeta\right\} \\
& =l-\omega t+M_{1} \lambda \int_{0}^{t}(t-\zeta) f(\zeta) d \zeta \\
& \leqslant l-\omega t+M_{1} \lambda \int_{0}^{t}\left(\frac{1}{\mu}-\zeta\right) f(\zeta) d \zeta, \quad \text { for all } 0 \leqslant t \leqslant \frac{1}{\mu} .
\end{aligned}
$$

By the generalized Gronwall inequality (see Hale [5]),

$$
\begin{aligned}
f(t) & \leqslant l-\omega t+M_{1} \lambda \int_{0}^{t}\left(\frac{1}{\mu}-\zeta\right)(l-\omega \zeta) e^{M_{1} \lambda / 2 \mu^{2}} d \zeta \\
& =l-\omega t+M_{1} \lambda e^{M_{1} \lambda / 2 \mu^{2}}\left(\frac{\omega}{\mu^{2}} t-\frac{\omega}{\mu} t^{2}+\frac{1}{3} \omega t^{3}\right)
\end{aligned}
$$

for all $0 \leqslant t \leqslant 1 / \mu$. In particular,

$$
f(1 / \mu) \leqslant(1 / 3) M_{1} \lambda e^{M_{1} \lambda / 2 \mu^{2}} \omega / \mu^{3},
$$

from which we deduce, using (2-28), (2-29),

$$
\frac{u\left(x\left(1 / \mu, \eta_{2}\right), 1 / \mu\right)-u\left(x\left(1 / \mu, \eta_{1}\right), 1 / \mu\right)}{x\left(1 / \mu, \eta_{2}\right)-x\left(1 / \mu, \eta_{1}\right)}<-3 \mu .
$$

Hence, for some $y_{2}>y_{1}$,

$$
\frac{u\left(y_{2}, 1 / \mu\right)-u\left(y_{1}, 1 / \mu\right)}{y_{2}-y_{1}}=-3 \mu .
$$

Let us summarize what we have obtained so far: If we suppose that $u(x, t) \in$ $C\left([0, \infty) ; H^{s}\right), s>3 / 2$,

$$
\frac{u\left(x_{2}, 0\right)-u\left(x_{1}, 0\right)}{x_{2}-x_{1}}=-\mu<0,
$$

$x_{2}>x_{1}$ and $\|u(x, 0)\|_{L^{2}} / \mu^{2} \leqslant \kappa($ defined by $(2-27),(2-28))$, then at time $t=1 / \mu$,

$$
\frac{u\left(y_{2}, t\right)-u\left(y_{1}, t\right)}{y_{2}-y_{1}}=-3 \mu, \text { for some } y_{2}>y_{1} \text {. }
$$

Recalling that $\|u(x, t)\|_{L^{2}}$ is constant for all $t \geqslant 0$, taking $1 / \mu$ as our initial time and repeating the above process, we arrive at

$$
\frac{u\left(z_{2}, 1 / \mu+1 / 3 \mu\right)-u\left(z_{1}, 1 / \mu+1 / 3 \mu\right)}{z_{2}-z_{1}}=-9 \mu,
$$

for some $z_{2}>z_{1}$. By indefinite iteration we conclude that as $t$ approaches $\mu^{-1}\left(1+1 / 3+1 / 3^{2}+\cdots\right)=\frac{3}{2} \mu^{-1},-\min _{x \in R} u_{x}(x, t)$ tends to $+\infty$, which contradicts our assumption that $u(x, t) \in C\left([0, \infty) ; H^{s}\right), s>3 / 2$. This completes the proof for equation (1-1). As mentioned in the preceding section, equations (1-2) and (1-9) are equivalent provided $u(x, t) \in C\left([0, T) ; H^{s}\right), s>3 / 2$; hence, we 
consider (1-9) to prove the assertion for (1-2). By virtue of $(2-13)$, we derive that for all $f \in L^{2}(R)$,

$$
\left|\int_{-\infty}^{\infty}\left\{G_{3}\left(x_{1}-y\right)-G_{3}\left(x_{2}-y\right)\right\} f(y) d y\right| \leqslant\left|x_{1}-x_{2}\right|\left\|G_{3 x}\right\|_{L^{2}}\|f\|_{L^{2}}
$$

and

$$
\left.(2-40)\left|\int_{-\infty}^{\infty}\left\{G_{3}\left(x_{1}-y\right)-G_{3}\left(x_{2}-y\right)\right\}\right| f(y)\right|^{2} d y|\leqslant| x_{1}-x_{2} \mid\left\|G_{3 x}\right\|_{L^{\infty}}\|f\|_{L^{2}}^{2}
$$

Using these inequalities and Lemma 2.3, and going through the same procedure as above, we can arrive at a similar conclusion for equation (1-9); we omit the details.

Seliger [10] also considered some model equations of nonlinear wave motions. However, the method in [10] is not applicable to our problem. The main reason is that $u_{x x}(x, t)$ may not be defined as an ordinary function under our assumption. Even if all the assumptions in [10] were met, a result analogous to Theorem 2.2 cannot be inferred from the result of [10]. Finally, we recall some known results on the solutions of (0-3) and (0-4). For a given initial function in $H^{s}, s>3 / 2,(0-3)$ and (0-4) have unique local solutions in $C\left([0, T) ; H^{s}\right), s>3 / 2$; see Kato [6, 7]. It is known that a local solution of $(0-4)$ in $\left([0, T) ; H^{s}\right)$ can be extended globally in time provided $s \geqslant 2$, while $(0-3)$ does not admit any global solution in $C\left([0, \infty) ; H^{s}\right), s>3 / 2$, unless the initial function is identically zero.

3. Existence of travelling wave solutions. We shall seek solutions of $(1-1),(1-2)$ in the form $\phi(x-c t), \phi \neq \equiv$ constant. For this purpose we consider the following equations with $c \neq \alpha$ :

$$
\begin{aligned}
& \phi-\frac{1}{c-\alpha} \mathbf{P}_{1} \phi=\frac{1}{2} \frac{1}{c-\alpha} \phi^{2}, \\
& \phi-\frac{c}{\alpha-c} \mathbf{P}_{2} \phi=\frac{1}{2} \frac{1}{c-\alpha} \phi^{2} .
\end{aligned}
$$

Let us suppose that $\mathbf{P}_{1}, \mathbf{P}_{2}$ are convolution operators with a kernel in $L^{1}(R)$ and $\phi(\cdot)$ is a solution of (3-1) (resp. (3-2)) in $L^{\infty}(R)$. Then, $\phi(x-c t)$ is a solution (in the distribution sense) of $(1-1)$ (resp. (1-2)) provided $u u_{x}$ is interpreted as $\left(\frac{1}{2} u^{2}\right)_{x}$. Therefore, we try to find solutions of (3-1), (3-2) under the following assumptions on $\mathbf{P}_{1}$ and $\mathbf{P}_{2}$ :

(3-3) $\hat{\mathbf{P}}_{i}(\xi), i=1,2$, are even, real-valued functions;

(3-4) $\hat{\mathbf{P}}_{i}(\xi) \in L^{1} \cup L^{2}$ and $d \hat{\mathbf{P}}_{i}(\xi) / d \xi \in L^{2}, i=1,2$;

(3-5) there exist $p>0$ and $s \neq 0, \hat{\mathbf{P}}_{1}(0)$, such that the set $\left\{\xi: \hat{\mathbf{P}}_{1}(\xi)=s\right\} \cap$ $\{2 \pi k / p: k=0,1,2, \ldots\}$ is not empty and consists of an odd number of points;

(3-6) there exist $p>0$ and $s \neq-1 / \sqrt{2 \pi}, 0, \hat{\mathbf{P}}_{2}(0)$, such that the set $\left\{\xi: \hat{\mathbf{P}}_{2}(\xi)=\right.$ $s\} \cap\{2 \pi k / p: k=0,1,2, \ldots\}$ is not empty and consists of an odd number of points.

THEOREM 3.1. Under assumptions (3-3) to (3-5) there are nonconstant, periodic solutions to $(3-1)$ for suitable $c \neq \alpha$.

TheOrem 3.2. Suppose $\alpha \neq 0$ and (3-3), (3-4) and (3-6) hold. Then (3-2) admits nonconstant periodic solutions for suitable $c \neq \alpha$. 
Proof of Theorem 3.1. Let us write (3-1) as

$$
\phi-\lambda \mathbf{P}_{1} \phi=\frac{1}{2} \lambda \phi^{2}
$$

where $\lambda$ is a parameter. By virtue of $(3-4)$ we can define a function $F(\cdot)$ such that $\hat{F}(\xi)=\hat{\mathbf{P}}_{1}(\xi)$; then $F$ is an even function in $L^{1}(R)$ (see [8]). Consequently, $\mathbf{P}_{1}$ is defined by

$$
\left(\mathbf{P}_{1} \phi\right)(x)=\int_{-\infty}^{\infty} F(x-y) \phi(y) d y, \quad \text { for all } \phi \in L^{\infty}(R)
$$

Next we define

$$
F_{p}(x)=\sum_{k=-\infty}^{\infty} F(x+k p)
$$

with $p$ which appeared in (3-5). Then the series converges absolutely at almost all $x$ and $F_{p}(x)$ is a $p$-periodic, even function. It is easy to see that $F_{p}(x)$ is integrable over $[0, p]$ and can be expanded in trigonometric functions:

$$
\begin{aligned}
F_{p}(x) & \sim \sum_{k=-\infty}^{\infty} \frac{\sqrt{2 \pi}}{p} \hat{\mathbf{P}}_{1}\left(\frac{2 \pi k}{p}\right) e^{i(2 \pi k x / p)} \\
& \sim \frac{\sqrt{2 \pi}}{p} \hat{\mathbf{P}}_{1}(0)+\sum_{k=1}^{\infty} \frac{2 \sqrt{2 \pi}}{p} \hat{\mathbf{P}}_{1}\left(\frac{2 \pi k}{p}\right) \cos \left(\frac{2 \pi k}{p} x\right)
\end{aligned}
$$

Next we define the function space

$$
\mathbf{S}_{p}=\left\{\sum_{k=0}^{\infty} a_{k} \cos \left(\frac{2 \pi k}{p} x\right): a_{k} \in R, \sum_{k=0}^{\infty}\left|a_{k}\right|^{2}\left\{1+\left(\frac{2 \pi k}{p}\right)^{2}\right\}<\infty\right\}
$$

equipped with the norm $\left(\sum_{k=0}^{\infty}\left|a_{k}\right|^{2}\left\{1+(2 \pi k / p)^{2}\right\}\right)^{1 / 2}$ and the corresponding inner product; then $\mathbf{S}_{p}$ is a real Hilbert space. We also define the operator $T_{p}$ by

$$
\left(T_{p} \phi\right)(x)=\int_{0}^{p} F_{p}(x-y) \phi(y) d y, \quad \text { for each } \phi \in \mathbf{S}_{p} .
$$

Then we have

LEMMA 3.3. $T_{p}$ is a compact, selfadjoint operator in $\mathbf{S}_{p}$ and has an eigenvalue $\sqrt{2 \pi} s$ of odd multiplicity (recall that $s$ and $p$ appeared in (3-5)).

Proof. Let $\phi=\sum_{k=0}^{\infty} a_{k} \cos (2 \pi k x / p) \in \mathbf{S}_{p}$. Then from (3-10) and (3-12) it follows that

$$
T_{p} \phi \sim \sum_{k=0}^{\infty} \sqrt{2 \pi} \hat{\mathbf{P}}_{1}\left(\frac{2 \pi k}{p}\right) a_{k} \cos \left(\frac{2 \pi k}{p} x\right)
$$


By the Riemann-Lebesgue Lemma, $\hat{\mathbf{P}}_{1}(2 \pi k / p)$ converges to 0 as $k$ tends to infinity and we obtain the estimate

$$
\begin{aligned}
& \sum_{k=0}^{\infty}\left|\hat{\mathbf{P}}_{1}\left(\frac{2 \pi k}{p}\right)\right|^{2}\left|a_{k}\right|^{2}\left\{1+\left(\frac{2 \pi k}{p}\right)^{2}\right\} \\
& \quad \leqslant \max _{k}\left|\hat{\mathbf{P}}_{1}\left(\frac{2 \pi k}{p}\right)\right|^{2} \sum_{k=0}^{\infty}\left|a_{k}\right|^{2}\left\{1+\left(\frac{2 \pi k}{p}\right)^{2}\right\},
\end{aligned}
$$

which implies that the infinite series of (3-13) converges absolutely for all $x$ and is equal to $\left(T_{p} \phi\right)(x)$ for all $x$. Now it is obvious that $T_{p}$ is a continuous mapping from $\mathbf{S}_{p}$ into itself and that $T_{p}$ is selfadjoint in $\mathbf{S}_{p}$. For each $m \geqslant 1$ the operator $T_{p, m}$, defined by

$$
T_{p, m} \phi=\sum_{k=0}^{m} \sqrt{2 \pi} \hat{\mathbf{P}}_{1}\left(\frac{2 \pi k}{p}\right) a_{k} \cos \left(\frac{2 \pi k}{p} x\right),
$$

for $\phi=\sum_{k=0}^{\infty} a_{k} \cos (2 \pi k x / p) \in \mathbf{S}_{p}$, is a compact operator from $\mathbf{S}_{p}$ into itself. Since the operator norm $\left\|T_{p}-T_{p, m}\right\|$ is bounded by $\sqrt{2 \pi} \max _{k \geqslant m+1}\left|\hat{\mathbf{P}}_{1}(2 \pi k / p)\right|$, we conclude that $\left\|T_{p}-T_{p, m}\right\|$ converges to 0 as $m$ tends to infinity. Thus, $T_{p}$ is also compact. Finally, combining (3-5) and (3-13), we deduce that $\sqrt{2 \pi} s$ is an eigenvalue of odd multiplicity in $\mathbf{S}_{p}$.

LEMMA 3.4. The mapping of $\phi \rightarrow \phi^{2}$ is a $C^{\infty}$-mapping from $\mathbf{S}_{p}$ into itself.

Proof. First we observe that that $S_{p}$ norm is equivalent to the $H^{1}(0, p)$ norm and $\mathbf{S}_{p}$ is a closed subspace of $H^{1}(0, p)$. Let

$$
\phi=\sum_{k=0}^{\infty} a_{k} \cos \left(\frac{2 \pi k}{p} x\right) \in \mathbf{S}_{p} \text { and } \phi_{m}=\sum_{k=0}^{m} a_{k} \cos \left(\frac{2 \pi k}{p} x\right) .
$$

From the identity $\cos \beta \cos \gamma=\frac{1}{2}\{\cos (\beta+\gamma)+\cos (\beta-\gamma)\}$, we infer that $\phi_{m}^{2} \in \mathbf{S}_{p}$ for each $m$. In the mean time it is known that if $f, g \in H^{1}(0, p)$, then $f g \in H^{1}(0, p)$ and

$$
\|f g\|_{H^{1}(0, p)} \leqslant \mathbf{M}\|f\|_{H^{1}(0, p)}\|g\|_{H^{1}(0, p)}
$$

holds with a constant $M>0$ independent of $f, g$. Therefore $\phi_{m}^{2}$ converges to $\phi^{2}$ in $H^{1}(0, p)$ as $m$ tends to infinity, and we conclude that $\phi^{2} \in \mathbf{S}_{p}$. Now it is easy to see that the mapping $\phi \rightarrow \phi^{2}$ is $C^{\infty}$ from $\mathbf{S}_{p}$ into itself.

With the aid of Lemmas 3.3 and 3.4, we can easily show that all the hypotheses in Theorem A of Westreich [11] are satisifed. Hence, it follows that $(1 / \sqrt{2 \pi} s, 0) \in R$ $\times \mathbf{S}_{p}$ is a bifurcation point for the equation

$$
\phi-\lambda T_{p} \phi=\frac{1}{2} \lambda \phi^{2} .
$$

In other words, for any given $\varepsilon>0$, there is a nonzero element $\phi_{\varepsilon}$ in $S_{p}$ and $\lambda_{\varepsilon} \in R$ such that $\left|\lambda_{\varepsilon}-1 / \sqrt{2 \pi} s\right|<\varepsilon,\left\|\phi_{\varepsilon}\right\|_{S_{p}}<\varepsilon$ and $\left(\lambda_{\varepsilon}, \phi_{\varepsilon}\right)$ satisfies (3-16). It remains to show that for sufficiently small $\varepsilon>0, \phi_{\varepsilon}$ cannot be a constant function. In fact, when $\phi_{\varepsilon} \equiv \rho$, for some constant, $\rho, 0<|\rho|<\varepsilon,(3-16)$ implies

$$
\rho-\lambda_{\varepsilon} \sqrt{2 \pi} \hat{\mathbf{P}}_{1}(0) \rho=\frac{1}{2} \lambda_{\varepsilon} \rho^{2} \text {. }
$$


If $\varepsilon$ is sufficiently small, $1-\lambda_{\varepsilon} \sqrt{2 \pi} \hat{\mathrm{P}}_{1}(0)$ is bounded away from zero by condition (3-5) and, consequently, (3-17) cannot hold for small $\rho \neq 0$. If $\phi \in \mathbf{S}_{p}$ then $\phi$ is a $p$-periodic, continuous function in $L^{\infty}(R)$ and the following holds:

$$
\int_{0}^{p} F_{p}(x-y) \phi(y) d y=\int_{-\infty}^{\infty} F(x-y) \phi(y) d y .
$$

Hence, a solution of (3-16) in $\mathbf{S}_{p}$ is also a solution of (3-1) by choosing suitable $c \neq \alpha$. This completes the proof of Theorem 3.1.

Proof of Theorem 3.2. Suppose $\alpha \neq 0$ and write (3-2) as

$$
\phi-\lambda \mathbf{P}_{2} \phi=-(\lambda+1) \phi^{2} / 2 \alpha,
$$

where $\lambda$ is a parameter. The above proof of Theorem 3.1 can be repeated to establish the existence of nonconstant periodic solutions to (3-2). The additional conditions that $s \neq-1 / \sqrt{2 \pi}$ and that $\alpha \neq 0$ are necessary to choose a constant $c$ corresponding to suitable $\lambda$, that is, $c \in R$ can be chosen so that $c /(\alpha-c)=\lambda$ when $\lambda \neq-1$, $\alpha \neq 0$.

As an application of the above results, we specialize on (0-1) and (0-2), which we rewrite as

$$
\begin{aligned}
& u_{t}+\alpha u_{x}+u u_{x}+\frac{\partial}{\partial x} \int_{-\infty}^{\infty} \Gamma(x-y) u(y, t) d y=0 \\
& u_{t}+\alpha u_{x}+u u_{x}-\frac{\partial}{\partial t} \int_{-\infty}^{\infty} \Gamma(x-y) u(y, t) d y=0
\end{aligned}
$$

where

$$
\Gamma(x)=A \frac{\partial^{2}}{\partial x^{2}} \frac{1}{\sqrt{x^{2}+B^{2}}} .
$$

This interpretation of $(0-1)$ and $(0-2)$ is admissible since the original model equation was derived in terms of the symbol of the operator (see [9]). Moreover, this form of the equations has an advantage in that the integral is well defined when $u(\cdot, t)$ is merely in $L^{\infty}$. Restricting our attention to the case $c>\alpha$, our claim for equation $(3-20)$ is:

THEOREM 3.4. Suppose $A \neq 0$ and $B>0$. Then (3-20) has nonconstant, periodic travelling wave solutions of the form $\phi(x-c t), c>\alpha$. If $A<0$, the amplitude of $\phi$ can be arbitrarily small (with $c-\alpha$ bounded away from zero). If $A>0$ and $c-\alpha>\varepsilon$ for some $\varepsilon>0$, then there is no nonconstant, periodic solution of the form $\phi(x-c t)$ with $\|\phi\|_{L^{\infty}} \leqslant \varepsilon / 2$.

Proof. We consider equation (3-1) with $\hat{\mathbf{P}}_{1}(\xi)=-A \sqrt{2 / \pi} \xi^{2} K_{0}(B|\xi|)$, where $K_{0}(\cdot)$ is the modified Bessel function of the second kind of order zero. It is known that $\xi^{2} K_{0}(|\xi|)$ is nonnegative for all $\xi$ and that it behaves like $-\xi^{2} \log |\xi|$ for small $\xi$, and $\sqrt{\pi / 2}|\xi|^{3 / 2} e^{-|\xi|}$ for large $\xi$. Moreover, $\max _{\xi \geqslant 0} \xi^{2} K_{0}(B|\xi|)$ occurs at a single point, which we denote by $\xi_{0} ; 1.55 / B<\xi_{0}<1.56 / B$. Accordingly, conditions (3-3), (3-4) are satisfied and we choose

$$
s_{0}=-A \sqrt{2 / \pi} \xi_{0}^{2} K_{0}\left(B \xi_{0}\right)
$$


and

$$
p_{0}=2 \pi k_{0} \xi_{0}, \text { for any fixed positive integer } k_{0},
$$

so that condition (3-5) is satisfied. In fact, the set $\left\{\xi: \hat{\mathbf{P}}_{1}(\xi)=s_{0}\right\} \cap\left\{2 \pi k / p_{0}\right.$ : $k=0,1,2, \ldots\}$ is a single point $\xi_{0}$ and, hence, the eigenvalue $\sqrt{2 \pi} s_{0}$ (or $\left.-2 A \xi_{0}^{2} K_{0}\left(B \xi_{0}\right)\right)$ is simple with an eigenfunction $\cos \left(\xi_{0} x\right)$. Here we can use a theorem of Crandall-Rabinowitz [4] to conclude that (3-1) has solutions

$$
\phi(x)=\varepsilon \cos \left(\xi_{0} x\right)+\varepsilon g(\varepsilon)
$$

with $c-\alpha=\sqrt{2 \pi} s_{0}+h(\varepsilon)$ for sufficiently small $\varepsilon>0$, where $g(\varepsilon)$ is a $c^{\infty}$ function of $\varepsilon$ into the complement of $\operatorname{span}\left\{\cos \left(\xi_{0} x\right)\right\}$ in $\mathbf{S}_{p_{0}}$ and $h(\varepsilon)$ is a $c^{\infty}$ function of $\varepsilon$ into $R$ such that $g(0)=0, h(0)=0$. When $A<0, s_{0}$ is positive by (3-22) and thus the first part of the theorem has been proved. Next let us consider the case $A>0$ which implies $s_{0}<0$. Since we are interested in solutions of the form $\phi(x-c t), c>\alpha$, we define a new function $\Psi$ by

$$
\Psi=-2(c-\alpha)+\phi
$$

and write (3-1) in terms of $\Psi$ :

$$
\Psi-\frac{1}{\alpha-c} \mathbf{P}_{1} \Psi=\frac{1}{2} \frac{1}{\alpha-c} \Psi^{2},
$$

where we have used the fact that $\mathbf{P}_{1}$ annihilates constant functions since $\hat{\mathbf{P}}_{1}(\xi)=$ $-A \sqrt{2 / \pi} \xi^{2} K_{0}(B|\xi|)$. Now we have $\alpha-c$ in place of $c-\alpha$, which is the only difference between (3-1) and (3-25). So we know that (3-25) has solutions $\varepsilon \cos \left(\xi_{0} x\right)+\varepsilon g(\varepsilon)$ with $\alpha-c=\sqrt{2 \pi} s_{0}+h(\varepsilon)$ for sufficiently small $\varepsilon>0$, where $g, h$ are the same functions as above. Hence we have obtained solutions $\phi(x)$ of $(3-1)$ in the form

$$
\phi(x)=2\left\{2 \mathbf{A} \xi_{0}^{2} K_{0}\left(\mathbf{B} \xi_{0}\right)-h(\varepsilon)\right\}+\varepsilon \cos \left(\xi_{0} x\right)+\varepsilon g(\varepsilon),
$$

with $c-\alpha=2 A \xi_{0}^{2} K_{0}\left(B \xi_{0}\right)-h(\varepsilon)$, which is positive when $A>0$ and $\varepsilon$ is sufficiently small. Finally, we suppose that $A>0, c-\alpha>\varepsilon>0$ and (3-20) has a nonconstant periodic solution $\phi(x-c t)$ in $L^{\infty}$ with period $p$. Then, using (3-18), we find that $\phi(x)$ satisfies

$$
\phi(x)-\frac{1}{c-\alpha} \int_{0}^{p} F_{p}(x-y) \phi(y) d y=\frac{1}{2(c-\alpha)} \phi^{2}(x)+M,
$$

where $M$ is a constant. Let us define

$$
\sigma(x)=\phi(x)-\frac{1}{p} \int_{0}^{p} \phi(y) d y
$$

and write (3-26) in terms of $\sigma(x)$ :

$$
\begin{aligned}
\sigma(x)- & \frac{1}{c-\alpha} \int_{0}^{p} F_{p}(x-y) \sigma(y) d y \\
& =\frac{1}{2(c-\alpha)} \sigma^{2}(x)+\frac{1}{p(c-\alpha)}\left(\int_{0}^{p} \phi(y) d y\right) \sigma(x)+\tilde{M}
\end{aligned}
$$


where $\tilde{M}$ is a constant and we have used the fact that $\int_{0}^{p} F_{p}(x-y) d y=0$ (notice that $\left.\hat{\mathbf{P}}_{1}(0)=0\right)$. Multiplying both sides of (3-28) by $\sigma(x)$ and integrating over $(0, p)$, we obtain

$$
\begin{aligned}
\int_{0}^{p} \sigma^{2}(x) d x & \leqslant \frac{1}{c-\alpha}\left\{\frac{1}{2}\|\sigma\|_{L^{\infty}}+\frac{1}{p}\left|\int_{0}^{p} \phi(y) d y\right|\right\} \int_{0}^{p} \sigma^{2}(x) d x \\
& \leqslant \frac{1}{c-\alpha} 2\|\phi\|_{L^{\infty}} \int_{0}^{p} \sigma^{2}(x) d x,
\end{aligned}
$$

since $\int_{0}^{p} \sigma(x) d x=0$ and

$$
\begin{aligned}
& \int_{0}^{p}\left(\int_{0}^{p} F_{p}(x-y) \phi(y) d y\right) \phi(x) d x \\
& \quad=\sqrt{2 \pi} p \hat{\mathbf{P}}_{1}(0) a_{0}^{2}+\sum_{k=1}^{\infty} \sqrt{2 \pi} \frac{p}{2} \hat{\mathbf{P}}_{1}\left(\frac{2 \pi k}{p}\right)\left(a_{k}^{2}+b_{k}^{2}\right) \\
& \quad=-\sum_{k=1}^{\infty} A p\left(\frac{2 \pi k}{p}\right)^{2} K_{0}\left(B \frac{2 \pi k}{p}\right)\left(a_{k}^{2}+b_{k}^{2}\right) \leqslant 0
\end{aligned}
$$

where

$$
\phi(x) \sim \sum_{k=0}^{\infty}\left(a_{k} \cos \frac{2 \pi k}{p} x+b_{k} \sin \frac{2 \pi k}{p} x\right) .
$$

Since $\phi$ is nonconstant, $\int_{0}^{p} \sigma^{2}(x) d x>0$ and hence, it follows from (3-29) that $\|\phi\|_{L^{\infty}} \geqslant(c-\alpha) / 2>\varepsilon / 2$. This concludes the proof of Theorem 3.4.

REMARK 3.5. We note that there are infinitely many $s, p$ such that $\left\{\xi: \hat{\mathbf{P}}_{1}(\xi)=s\right\}$ $\cap\{2 \pi k / p: k=0,1,2, \ldots\}$ is a single point; we could have chosen other points rather than $s_{0}, p_{0}$ in the above proof. Now we elaborate on this fact. When $\mathbf{P}_{1}(\xi)=-A \sqrt{2 / \pi} \xi^{2} K_{0}(B|\xi|), \hat{\mathbf{P}}_{1}(\xi)$ is monotonically increasing on $\left[0, \xi_{0}\right]$ and monotonically decreasing on $\left[\xi_{0}, \infty\right)$ or vice versa depending on $A$. Therefore, it is easy to show that the set $\{s: \exists p>0$, such that $s, p$ satisfy (3-5) $\}$ is dense in the interval $\left(0, s_{0}\right)$ (or $\left.\left(s_{0}, 0\right)\right)$. Suppose $A<0$. Let $s_{1}$ be any number in $\left(0, s_{0}\right)$ and $\xi_{1}<\xi_{2}$ be two roots of $\hat{\mathbf{P}}_{1}(\xi)=s_{1}$. If (3-5) is not satisfied by $s_{1}$, we infer that if $\xi_{1}=2 \pi k_{1} / p$ for some $p>0$ and integer $k_{1}>0$, then there is a positive integer $k_{2}$ so that $\xi_{2}=2 \pi k_{2} / p$. Suppose this is the case. Set $s_{1}^{*}=s_{1}-\delta>0$, for small $\delta>0$. Then $\xi_{1}=2 \pi k_{1} / p, \xi_{2}=2 \pi k_{2} / p$ for some $p>0$ and integers $k_{1}, k_{2}>0$. Let $\xi_{1}^{*}<\xi_{2}^{*}$ be roots of $\hat{\mathbf{P}}_{1}(\xi)=s_{1}^{*}$ and choose $p^{*}$ so that $\xi_{1}^{*}=2 \pi k_{1} / p^{*}$. Obviously, $\xi_{1}^{*}<\xi_{1}<\xi_{2}<$ $\xi_{2}^{*}$. Thus, $p^{*}>p$ and $2 \pi k_{2} / p^{*}<2 \pi k_{2} / p=\xi_{2}$. If $\delta$ is sufficiently small, then $p^{*}, \xi_{2}^{*}$ are so close to $p, \xi_{2}$, respectively, that there is no integer $k_{2}^{*}>0$ such that $\xi_{2}^{*}=2 \pi k_{2}^{*} / p^{*}>2 \pi k_{2} / p=\xi_{2}$. Hence, $s_{1}-\delta$ may be taken to satisfy (3-5). The proof for the case $A>0$ is similar.

Now we turn our attention to equation (3-21), for which our assertion is:

TheOREM 3.6. Suppose $\alpha>0, A \neq 0$ and $B>0$. Then (3-21) has nontrivial, periodic travelling wave solutions of the form $\phi(x-c t), c>\alpha$. If $A<0$, the amplitude of $\phi$ can be arbitrarily small (with $c-\alpha$ bounded away from zero). If $A>0$ and $c-\alpha>\varepsilon$, then there is no nonconstant, periodic solution of the form $\phi(x-c t)$ with $\|\phi\|_{L^{\infty}} \leqslant \varepsilon / 2$. 
Proof. We consider equation (3-2) with $\hat{\mathbf{P}}_{2}(\xi)=A \sqrt{2 / \pi} \xi^{2} K_{0}(B|\xi|)$, where the bifurcation parameter $\lambda$ is related to $c$ by $c /(\alpha-c)=\lambda$. Hence, $c>\alpha$ holds if and only if $\lambda<-1$. Suppose $A<0$. With the aid of Remark 3.5 we can find $\xi_{1}>0$, $p_{1}>0$ and integer $k_{1}>0$ such that $\xi_{1}=2 \pi k_{1} / p_{1},-1<2 A \xi_{1}^{2} K_{0}\left(B \xi_{1}\right)<0$ and such that $s_{1}=A \sqrt{2 / \pi} \xi_{1}^{2} K_{0}\left(B \xi_{1}\right), p_{1}$ satisfy condition (3-6). By repetition of the previous argument, we derive that (3-2) has solutions

$$
\varepsilon \cos \left(\xi_{1} x\right)+\varepsilon g_{1}(\varepsilon),
$$

with $\lambda=1 / \sqrt{2 \pi} s_{1}+h_{1}(\varepsilon)$ for sufficiently small $\varepsilon>0$, where $g_{1}(\varepsilon)$ is a $c^{\infty}$ mapping of $\varepsilon$ into the complement of $\left\{\cos \left(\xi_{1} x\right)\right\}$ in $S_{p_{1}}$ and $h_{1}(\varepsilon)$ is a $c^{\infty}$-mapping of $\varepsilon$ into $R$ such that $g_{1}(0)=0, h_{1}(0)=0$. Now we can choose $c>\alpha$ such that $c /(\alpha-c)=\lambda<$ -1 . For the case $A>0$, we again use Remark 3.5 after introducing $\Psi=2 \alpha /(\lambda+1)$ $+\phi$ as in the proof of Theorem 3.4. The last assertion is also proved as before and we omit the details.

REMARK 3.7. It is well known that the K-dV equation admits both solitary wave solutions and periodic travelling wave solutions. For equations (3-20), (3-21), the existence of solitary wave solutions is not known.

Acknowledgement. I am very grateful to Professor John Nohel for his enthusiastic support.

\section{REFERENCES}

1. T. B. Benjamin, Lectures on nonlinear wave motion, Lectures in Appl. Math., Vol. 15 (A. Newell, ed.), Amer. Math. Soc., Providence, R. I., 1974, pp. 3-47.

2. T. B. Benjamin, J. L. Bona and J. J. Mahony, Model equations for long waves in nonlinear dispersive systems, Philos. Trans. Roy. Soc. London Ser. A 272 (1972), 47-78.

3. J. L. Bona, On solitary waves and their role in the evolution of long waves, Applications of Nonlinear Analysis in the Physical Sciences (H. Amann, N. Bazley and K. Kirchgässner, ed.), Pitman, London, 1981, pp. 183-205.

4. M. Crandall and P. Rabinowitz, Bifurcation from single eigenvalues, J. Funct. Anal. 8 (1971), $321-340$.

5. J. Hale, Ordinary differential equations, Wiley, New York, 1969.

6. T. Kato, Quasi-linear equations of evolution with application in partial differential equations, Lecture Notes in Math., vol. 448, Springer-Verlag, Berlin, 1975, pp. 25-70.

7. __ On the Korteweg-de Vries equations, Manuscripta Math. 28 (1979), 89-99.

8. J. Kim, Solutions to the equations of one-dimensional viscoelasticity in BV, SIAM J. Math. Anal. 14 (1983), 684-695.

9. S. Leibovich, Weakly nonlinear waves in rotating fluids, J. Fluid Mech. 42 (1970), 802-822.

10. R. L. Seliger, A note on the breaking waves, Proc. Roy. Soc. London Ser. A 303 (1968), 493-496.

11. D. Westreich, Bifurcation at eigenvalues of odd multiplicity, Proc. Amer. Math. Soc. 41 (1973), 609-614.

Department of Mathematics, Virginia Polytechnic Institute and State University, BlacksBURG, VIRGINIA 24061 